\title{
Lens Design: A New Approach ${ }^{1}$ Orestes N. Stavroudis
}

(February 18, 1959)

\begin{abstract}
This paper describes a new method of defining the total aberrations of an optical system and its application to lens design. A system of partial differential equations defining optical image formation is written in the form of 4 by 4 matrices and is applied to the derivation of lens aberrations. It is shown that the form of the matrices facilitates the computation of these aberrations and that equations of condition on the lens parameters can be derived. The use of this method is illustrated through its application to a simple, well-known problem.
\end{abstract}

\section{Introduction}

In the course of designing a lens, one invariably computes aberration functions whose magnitudes reflect qualitatively and quantitatively the performance of an optical system. Unfortunately these quantities are rather difficult to calculate, usually requiring a long program of ray tracing, and, more importantly, are not readily correlated with the lens parameters. Usually a lens designer must rely on his experience when determining whether a change in a certain curvature, thickness, or index of refraction will affect a given aberration.

In this paper a method of attack will be described which will at least mitigate these difficulties. The application of this method results in the definition of a set of aberrations together with a systematic scheme for their computation expressed as equations in which the system parameters appear. Yet the method is sufficiently flexible to meet the special requirements of any lens. For example, one system of aberrations can be defined for use with a copy lens and a second system can be constructed for application to an afocal system.

Consider an optical system consisting of a system of lenses and fixed reference planes in object and image space which are not necessarily object and image planes. Right-handed coordinate systems are chosen in object and image space, the coordinates of which are designated $x, y, z$, and $x^{\prime}, y^{\prime}, z^{\prime}$, respectively. We take the positive $z$ and $z^{\prime}$ axes in the general direction of ray propagation and $x-y$ plane and $x^{\prime}-y^{\prime}$ plane coincident with the object and image reference planes. Let the reduced direction cosines of a ray in object and image space be $\xi, \eta, \zeta$ and $\xi^{\prime}, \eta^{\prime}, \zeta^{\prime}$, where $\xi^{2}+{ }^{2} \eta+\zeta^{2}=N^{2}, \xi^{\prime 2}+\eta^{\prime 2}+\zeta^{\prime 2}=N^{\prime 2}, N$ and $N^{\prime}$ being the indices of refraction of object and image space

Associated with each ray passing through the optical system are two sets of four independent coordinates, $x, y, \xi, \eta$, and $x^{\prime}, y^{\prime}, \xi^{\prime}, \eta^{\prime}$; for a given optical system either set completely determines a ray. In an obvious way a given optical system can be said to define $x^{\prime}, y^{\prime}, \xi^{\prime}$, and $\eta^{\prime}$ as functions of $x, y, \xi$, and $\eta$. In what follows we tacitly assume that in any optical system these four functions are continuous and possess continuous first-partial derivatives.

Suppose every ray emanating from an object point converges to a single point on the image plane. Then $x^{\prime}$ and $y^{\prime}$, considered as functions of the object space variables, are unaffected by a change of $\xi$ and $\eta$. Thus $x^{\prime}$ and $y^{\prime}$ are independent of $\xi$ and $\eta$ and

$$
x_{\xi}^{\prime}=x_{\eta}^{\prime}=y_{\xi}^{\prime}=y_{\eta}^{\prime}=0
$$

where the subscripts denote partial derivatives. The converse is also true; if there exists a pair of values for $x$ and $y$ for which (1) is satisfied, then all rays emanating from the point $(x, y)$ in object space converge to a single point $\left(x^{\prime}, y^{\prime}\right)$ in image space. Thus the four equations of (1)

1 This paper contains work done at the Imperial College of Science and Technology and included in a thesis submitted to the University of London in partial fulfillment of the requirements for the Ph. D. degree. The work was supported in part by the U.S. Air Force. 
provide a necessary and sufficient condition for a point to be imaged perfectly. By a similar argument it can be seen that in a region where (1) is satisfied a necessary and sufficient condition for a lens to be free from distortion is that

$$
x_{x}^{\prime}=y_{y}^{\prime}=m, \quad x_{y}^{\prime}=y_{x}^{\prime}=0,
$$

where $m$ is a constant and represents the magnification of the system.

These two sets of equations provide conditions assuring perfect, distortion-free image formation. By the same token they may be taken as measures of the failure of an optical system to achieve perfection; that is to say, they are measures of the aberrations of the system. What follows is an elaboration of this basic idea.

\section{The Fundamental Optical Invariant}

The functional relationship described in the preceding section is a general transformation of which optical image formation is a special case. It is desirable therefore to impose restrictions on the functions; to exclude from our attention those relationships that do not apply to optical systems. There are several ways in which this can be done. For the purposes of this study the most convenient method is through the use of Herzberger's fundamental optical invariant. This leads to a system of partial differential equations which must be satisfied by the functions $x^{\prime}, y^{\prime}, \xi^{\prime}$, and $\eta^{\prime}$ if they are to describe the image formation of an optical system. Following Herzberger [1] these are written,

$$
\left.\begin{array}{l}
x_{x}^{\prime} \xi_{y}^{\prime}+y_{x}^{\prime} \eta_{y}^{\prime}-x_{y}^{\prime} \xi_{x}^{\prime}-y_{y}^{\prime} \eta_{x}^{\prime}=0 \\
x_{x}^{\prime} \xi_{\xi}^{\prime}+y_{x}^{\prime} \eta_{\xi}^{\prime}-x_{\xi}^{\prime} \xi_{x}^{\prime}-y_{\xi}^{\prime} \eta_{x}^{\prime}=1 \\
x_{x}^{\prime} \xi_{\eta}^{\prime}+y_{x}^{\prime} \eta_{\eta}^{\prime}-x_{\eta}^{\prime} \xi_{x}^{\prime}-y_{\eta}^{\prime} \eta_{x}^{\prime}=0 \\
x_{y}^{\prime} \xi_{\xi}^{\prime}+y_{y}^{\prime} \eta_{\xi}^{\prime}-x_{\xi}^{\prime} \xi_{y}^{\prime}-y_{\xi}^{\prime} \eta_{y}^{\prime}=0 \\
x_{y}^{\prime} \xi_{\eta}^{\prime}+y_{y}^{\prime} \eta_{\eta}^{\prime}-x_{\eta}^{\prime} \xi_{y}^{\prime}-y_{\eta}^{\prime} \eta_{y}^{\prime}=1 \\
x_{\xi}^{\prime} \xi_{\eta}^{\prime}+y_{\xi}^{\prime} \eta_{\eta}^{\prime}-x_{\eta}^{\prime} \xi_{\xi}^{\prime}-y_{\eta}^{\prime} \eta_{\xi}^{\prime}=0 .
\end{array}\right\}
$$

It is particularly convenient to express these in matrix form as

where

$$
\bar{X} J X=J
$$

$$
\begin{gathered}
X=\left(\begin{array}{rrrr}
x_{x}^{\prime} & x_{y}^{\prime} & x_{\xi}^{\prime} & x_{\eta}^{\prime} \\
y_{x}^{\prime} & y_{y}^{\prime} & y_{\xi}^{\prime} & y_{\eta}^{\prime} \\
\xi_{x}^{\prime} & \xi_{y}^{\prime} & \xi_{\xi}^{\prime} & \xi_{\eta}^{\prime} \\
\eta_{x}^{\prime} & \eta_{y}^{\prime} & \eta_{\xi}^{\prime} & \eta
\end{array}\right), \\
J=\left(\begin{array}{rrrr}
0 & 0 & 1 & 0 \\
0 & 0 & 0 & 1 \\
-1 & 0 & 0 & 0 \\
0 & -1 & 0 & 0
\end{array}\right),
\end{gathered}
$$

and $\bar{X}$ represents the transpose of $X$.

It is important to note that $X$ is a Jacobian matrix. An interesting corollary to (4) is that

$$
|X|=1
$$

or, in words, the Jacobian of the transformation representing optical image formation is equal to unity. 
It is interesting to note that this corollary is equivalent to a theorem proven by Straubel [2] as long ago as 1902. He applied this to several problems including photometry. More recently Toraldo di Francia [3] used this theorem in describing a generalization of geometric optics.

The $X$-matrix is particularly useful. First of all its elements consist of the partial derivatives of image space variables with respect to object space variables and therefore can be said to represent the geometrical aberrations of an optical system. Moreover, it appears as an indeterminate in the equations derived from the fundamental optical invariant. Finally it can be decomposed into a product of $X$-matrices each representing a component of the lens. Thus, if we consider an optical system consisting of two lenses arranged so that the image plane of the first coincides with the object plane of the second then

$$
X=X_{2} X_{1}
$$

where $X_{1}$ is the $X$-matrix of the first lens, $X_{2}$ of the second and $X$ that of the composite optical system.

The proof of (6) follows at once from the properties of Jacobian matrices and the rules for differentiating a function of a function. Let the first lens be defined by the four functions $\bar{x}, \bar{y}$, $\bar{\xi}$, and $\bar{\eta}$ of $x, y, \xi$, and $\eta$ and let the second be defined by $x^{\prime}, y^{\prime}, \xi^{\prime}$, and $\eta^{\prime}$ of $\bar{x}, \bar{y}, \zeta$, and $\bar{\eta}$. The composite lens is then defined by $x^{\prime}, y^{\prime}, \xi^{\prime}$, and $\eta^{\prime}$ as functions of $x, y, \xi, \eta$. Then differentiating

Then

$$
\begin{aligned}
& x_{x}^{\prime}=x_{x}^{\prime} \bar{x}_{x}+y_{\bar{y}}^{\prime} \bar{y}_{x}+x_{\bar{\xi}}^{\prime} \xi_{x}+x_{\bar{\eta}}^{\prime} \bar{\eta}_{x} \\
& y_{x}^{\prime}=y_{\bar{x}}^{\prime} \bar{x}_{x}+y_{y}^{\prime} \bar{y}_{x}+y_{\bar{\xi}}^{\prime} \xi_{x}+y_{\bar{\eta}}^{\prime} \bar{\eta}_{x}, \text { etc. }
\end{aligned}
$$

$$
\begin{aligned}
& X=\left(\begin{array}{cccc}
x_{x}^{\prime} & x_{y}^{\prime} & x_{\xi}^{\prime} & x_{\eta}^{\prime} \\
y_{x}^{\prime} & y_{y}^{\prime} & y_{\xi}^{\prime \prime} & y_{\eta}^{\prime} \\
\xi_{x}^{\prime} & \xi_{y}^{\prime} & \xi_{\xi}^{\prime} & \xi_{\eta}^{\prime} \\
\eta_{z}^{\prime} & \eta_{y}^{\prime} & \eta_{\xi}^{\prime} & \eta_{\eta}^{\prime}
\end{array}\right)=\left(\begin{array}{cccc}
x_{\bar{x}}^{\prime} & x_{\bar{y}}^{\prime} & x_{\bar{\xi}}^{\prime} & x_{\bar{\eta}}^{\prime} \\
y_{\bar{x}}^{\prime} & y_{\bar{y}}^{\prime} & y_{\bar{\xi}}^{\prime} & y_{\bar{\eta}}^{\prime} \\
\xi_{\bar{x}}^{\prime} & \xi_{y}^{\prime} & \xi_{\bar{\xi}}^{\prime} & \xi_{\bar{\eta}}^{\prime} \\
\eta_{\bar{x}}^{\prime} & \eta_{\bar{y}}^{\prime} & \eta_{\bar{\xi}}^{\prime} & \eta_{\bar{\eta}}^{\prime}
\end{array}\right)\left(\begin{array}{llll}
\bar{x}_{x} & \bar{x}_{y} & \bar{y}_{\xi} & \bar{x}_{\eta} \\
\bar{y}_{x} & \bar{y}_{y} & \bar{y}_{\xi} & \bar{y}_{\eta} \\
\bar{\xi}_{x} & \bar{\xi}_{y} & \bar{\xi}_{\xi} & \bar{\xi}_{\eta} \\
\bar{\eta}_{x} & \bar{\eta}_{y} & \bar{\eta}_{\xi} & \bar{\eta}_{\eta}
\end{array}\right) \\
& =X_{2} X_{1} .
\end{aligned}
$$

Clearly this can be extended to lenses consisting of any number of components.

\section{Transformation of the X-Matrix}

So far we have been dealing with the variables $x, y$, $\xi$, and $\zeta$. It is convenient to apply a transformation, $\mathbf{T}$, mapping these variables into a new system of coordinates, say $\alpha, \beta, \gamma$, and $\delta$, given formally by writing the latter as continuously differentiable functions of the former. The same transformation is applied to both the object and image space variables.

If we designate the $M$-matrix by

then

$$
M=\left(\begin{array}{cccc}
\alpha_{\alpha}^{\prime} & \alpha_{\beta}^{\prime} & \alpha_{\gamma}^{\prime} & \alpha_{\delta}^{\prime} \\
\beta_{\alpha}^{\prime} & \beta_{\beta}^{\prime} & \beta_{\gamma}^{\prime} & \beta_{\delta}^{\prime} \\
\gamma_{\alpha}^{\prime} & \gamma_{\beta}^{\prime} & \gamma_{\gamma}^{\prime} & \gamma_{\delta}^{\prime} \\
\delta_{\alpha}^{\prime} & \delta_{\beta}^{\prime} & \delta_{\gamma}^{\prime} & \delta_{\delta}^{\prime}
\end{array}\right),
$$

where

$$
M=A^{\prime} X A^{-1} \text {, }
$$

$$
A=\left(\begin{array}{cccc}
\alpha_{x} & \alpha_{y} & \alpha_{\xi} & \alpha_{\eta} \\
\beta_{x} & \beta_{y} & \beta_{\xi} & \beta_{\eta} \\
\gamma_{x} & \gamma_{y} & \gamma_{\xi} & \gamma_{\eta} \\
\delta_{x} & \delta_{y} & \delta_{\xi} & \delta_{\eta}
\end{array}\right)
$$


$A^{\prime}$ represents the same matrix but in image-space variables. Applying this transformation to equation (4) we have

which reduces to

$$
\left(\bar{A}^{-1} \bar{X} \bar{A}^{\prime}\right) \bar{A}^{\prime-1} J A^{\prime-1}\left(A^{\prime} X A^{-1}\right)=\bar{A}^{-1} J A^{-1},
$$

$$
\bar{M}\left(\bar{A}^{\prime-1} J A^{\prime-1}\right) M=\left(\bar{A}^{\prime-1} J A^{-1}\right) .
$$

If the transformation, $\mathbf{T}$, is restricted by the equations

$$
\bar{A} J A=J, \quad \bar{A}^{\prime} J A^{\prime}=J,
$$

then (8) becomes

$$
\bar{M} J M=J,
$$

which is identical in form to (4) except that the variables are changed from $x, y, \xi$, and $\eta$ to $\alpha, \beta, \gamma$, and $\delta$. Thus we can change our variables in any way that is convenient, provided that $(9)$ is satisfied, without adding to the complexity of the fundamental optical invariant.

\section{Rotational Symmetric Systems}

In the case of rotationally symmetric systems we are in a position to make a particularly advantageous choice of one of the new variables. In such a system the skewness invariant holds and

$$
p=x^{\prime} \eta^{\prime}-y^{\prime} \xi^{\prime}=x \eta-y \xi
$$

By letting $\gamma=x \eta-y \xi=p$, and $\gamma^{\prime}=x^{\prime} \eta^{\prime}-y^{\prime} \xi^{\prime}$, the $M$-matrix degenerates to

$$
M=\left(\begin{array}{cccc}
\alpha_{\alpha}^{\prime} & \alpha_{\beta}^{\prime} & \alpha_{p}^{\prime} & \alpha_{\delta}^{\prime} \\
\beta_{\alpha}^{\prime} & \beta_{\beta}^{\prime} & \beta_{p}^{\prime} & \beta_{\delta}^{\prime} \\
0 & 0 & 1 & 0 \\
\delta_{\alpha}^{\prime} & \delta_{\beta}^{\prime} & \delta_{p}^{\prime} & \delta_{\delta}^{\prime}
\end{array}\right)
$$

When this is substituted into (10) we obtain the transformed fundamental optical invariant

$$
\left.\begin{array}{c}
\alpha_{\alpha}^{\prime}=1+\beta_{p}^{\prime} \delta_{\alpha}^{\prime}-\beta_{\alpha}^{\prime} \delta_{p}^{\prime} \\
\alpha_{\beta}^{\prime}=\beta_{p}^{\prime} \delta_{\beta}^{\prime}-\beta_{\beta}^{\prime} \delta_{p}^{\prime} \\
\alpha_{\delta}^{\prime}=\beta_{p}^{\prime} \delta_{\delta}^{\prime}-\beta_{\delta}^{\prime} \delta_{p}^{\prime} \\
\beta_{\alpha}^{\prime} \delta_{\beta}^{\prime}-\beta_{\beta}^{\prime} \delta_{\alpha}^{\prime}=0 \\
\beta_{\alpha}^{\prime} \delta_{\delta}^{\prime}-\beta_{\delta}^{\prime} \delta_{\alpha}^{\prime}=0 \\
\beta_{\beta}^{\prime} \delta_{\delta}^{\prime}-\beta_{\delta}^{\prime} \delta_{\beta}^{\prime}=1 .
\end{array}\right\}
$$

We may consider the first two equations of (12) as a linear, homogeneous, simultaneous algebraic system in $\beta_{\alpha}^{\prime}$ and $\delta_{\alpha}^{\prime}$. A necessary and sufficient condition for a non-trivial solution to exist is that the determinant of coefficients must vanish identically. However, the third equation of (12) shows that this determinant is identical to a non-zero constant. We conclude that $\beta_{\alpha}^{\prime}=\delta_{\alpha}^{\prime}=0$. Therefore the first equation of (11) reduces to $\alpha_{\alpha}^{\prime}=1$. The remaining equations of the transformed fundamental optical invariant become

$$
\left.\begin{array}{c}
\alpha_{\beta}^{\prime}=\beta_{p}^{\prime} \delta_{\beta}^{\prime}-\beta_{\beta}^{\prime} \delta_{p}^{\prime} \\
\alpha_{\delta}^{\prime}=\beta_{p}^{\prime} \delta_{\delta}^{\prime}-\beta_{\delta}^{\prime} \delta_{p}^{\prime}
\end{array}\right\}
$$


and

$$
\beta_{\beta}^{\prime} \delta_{\delta}^{\prime}-\beta_{\delta}^{\prime} \delta_{\beta}^{\prime}=1
$$

Solving (13) for $\beta_{p}^{\prime}$ and $\delta_{p}^{\prime}$, using (14), we obtain equivalent expressions

$$
\left.\begin{array}{l}
\beta_{p}^{\prime}=\beta_{\beta}^{\prime} \alpha_{\delta}^{\prime}-\beta_{\delta}^{\prime} \alpha_{\beta}^{\prime} \\
\delta_{p}^{\prime}=\delta_{\beta}^{\prime} \alpha_{\delta}^{\prime}-\delta_{\delta}^{\prime} \alpha_{\beta}^{\prime} .
\end{array}\right\}
$$

Thus, under the assumption of rotational symmetry the $M$-matrix reduces to

$$
M=\left(\begin{array}{cccc}
1 & \alpha_{\beta}^{\prime} & \alpha_{p}^{\prime} & \alpha_{\delta}^{\prime} \\
0 & \beta_{\beta}^{\prime} & \beta_{p}^{\prime} & \beta_{\delta}^{\prime} \\
0 & 0 & 1 & 0 \\
0 & \delta_{\beta}^{\prime} & \delta_{p}^{\prime} & \delta_{\delta}^{\prime}
\end{array}\right)
$$

By applying (15) this can be factored into

$$
M=\left(\begin{array}{cccc}
1 & 0 & 0 & 0 \\
0 & \beta_{\beta}^{\prime} & 0 & \beta_{\delta}^{\prime} \\
0 & 0 & 1 & 0 \\
0 & \delta_{\beta}^{\prime} & 0 & \delta_{\delta}^{\prime}
\end{array}\right)\left(\begin{array}{cccc}
1 & \alpha_{\beta}^{\prime} & \alpha_{p}^{\prime} & \alpha_{\delta}^{\prime} \\
0 & 1 & \alpha_{\delta}^{\prime} & 0 \\
0 & 0 & 1 & 0 \\
0 & 0 & -\alpha_{\beta}^{\prime} & 1
\end{array}\right) .
$$

\section{Further Simplification}

Suppose we have a rotationally-symmetric optical system defined by the functions $\alpha^{\prime}$, $\beta^{\prime}$ and $\delta^{\prime}$ of $\alpha, \beta, p$ and $\delta$, consisting of two components, the first defined by $\alpha^{\prime}, \beta^{\prime}$, and $\delta^{\prime}$ of $\bar{\alpha}, \bar{\beta}, \bar{p}$, and $\bar{\delta}$ and the second by $\bar{\alpha}, \bar{\beta}$, and $\bar{\delta}$ of $\alpha, \beta, p$ and $\delta$. Of course $p$ and $\bar{p}$ are identical numerically but play different logical roles which must be kept distinct. As indicated in (6),

$$
\left(\begin{array}{cccc}
1 & \alpha_{\beta}^{\prime} & \alpha_{p}^{\prime} & \alpha_{\delta}^{\prime} \\
0 & \beta_{\beta}^{\prime} & \beta_{p}^{\prime} & \beta_{\delta}^{\prime} \\
0 & 0 & 1 & 0 \\
0 & \delta_{\beta}^{\prime} & \delta_{p}^{\prime} & \delta_{\delta}^{\prime}
\end{array}\right)=\left(\begin{array}{cccc}
1 & \alpha_{\bar{\beta}}^{\prime} & \alpha_{\bar{p}}^{\prime} & \alpha_{\bar{\delta}}^{\prime} \\
0 & \beta_{\bar{\beta}}^{\prime} & \beta_{\bar{p}}^{\prime} & \beta_{\bar{\delta}}^{\prime} \\
0 & 0 & 1 & 0 \\
0 & \delta_{\bar{\beta}}^{\prime} & \delta_{\bar{p}}^{\prime} & \delta_{\bar{\delta}}^{\prime}
\end{array}\right)\left(\begin{array}{cccc}
1 & \bar{\alpha}_{\beta} & \bar{\alpha}_{p} & \bar{\alpha}_{\delta} \\
0 & \bar{\beta}_{\beta} & \bar{\beta}_{p} & \bar{\beta}_{\delta} \\
0 & 0 & 1 & 0 \\
0 & \bar{\delta}_{\beta} & \bar{\delta}_{p} & \bar{\delta}_{\delta}
\end{array}\right) .
$$

By interchanging the two right columns and the two bottom rows of each matrix in (17) we obtain

$$
\left(\begin{array}{cccc}
1 & \alpha_{\beta}^{\prime} & \alpha_{\delta}^{\prime} & \alpha_{p}^{\prime} \\
0 & \beta_{\beta}^{\prime} & \beta_{\delta}^{\prime} & \beta_{p}^{\prime} \\
0 & \delta_{\beta}^{\prime} & \delta_{\delta}^{\prime} & \delta_{p}^{\prime} \\
0 & 0 & 0 & 1
\end{array}\right)=\left(\begin{array}{cccc}
1 & \alpha_{\bar{\beta}}^{\prime} & \alpha_{\bar{\delta}}^{\prime} & \alpha_{\bar{p}}^{\prime} \\
0 & \beta_{\bar{\beta}}^{\prime} & \beta_{\bar{\delta}}^{\prime} & \beta_{\bar{p}}^{\prime} \\
0 & \delta_{\bar{\beta}}^{\prime} & \delta_{\bar{\delta}}^{\prime} & \delta_{\bar{p}}^{\prime} \\
0 & 0 & 0 & 1
\end{array}\right)\left(\begin{array}{cccc}
1 & \bar{\alpha}_{\beta} & \bar{\alpha}_{\delta} & \bar{\alpha}_{p} \\
0 & \bar{\beta}_{\beta} & \bar{\beta}_{\delta} & \bar{\beta}_{p} \\
0 & \bar{\delta}_{\beta} & \bar{\delta}_{\delta} & \bar{\delta}_{p} \\
0 & 0 & 0 & 1
\end{array}\right) .
$$

Now let

$$
\begin{aligned}
& A^{\prime}=\left(\alpha_{\beta}^{\prime}, \alpha_{\delta}^{\prime}\right), \quad B^{\prime}=\left(\alpha_{p}^{\prime}\right), \\
& C^{\prime}=\left(\begin{array}{c}
\beta_{\beta}^{\prime} \beta_{\delta}^{\prime} \\
\delta_{\beta}^{\prime} \delta_{\delta}^{\prime}
\end{array}\right), \quad D^{\prime}=\left(\begin{array}{c}
\beta_{p}^{\prime} \\
\delta_{p}^{\prime}
\end{array}\right) \text {, }
\end{aligned}
$$

with $\bar{A}, \bar{B}, \bar{C}, \bar{D}$ and $A, B, C, D$ defined similarly. Then, by partitioning the matrices of (18) we obtain 


$$
\left(\begin{array}{ccc}
1 & A^{\prime} & B^{\prime} \\
e & C^{\prime} & D^{\prime} \\
0 & f & 1
\end{array}\right)=\left(\begin{array}{ccc}
1 & \bar{A} & \bar{B} \\
e & \bar{C} & \bar{D} \\
0 & f & 1
\end{array}\right)\left(\begin{array}{ccc}
1 & A & B \\
e & C & D \\
0 & f & 1
\end{array}\right)
$$

where $e=\left(\begin{array}{l}0 \\ 0\end{array}\right)$ and $f=(0,0)$. Then from (19) we can obtain

$$
C^{\prime}=\bar{C} C
$$

or

$$
\left(\begin{array}{ll}
\beta_{\beta}^{\prime} & \beta_{\delta}^{\prime} \\
\delta_{\beta}^{\prime} & \delta_{\delta}^{\prime}
\end{array}\right)=\left(\begin{array}{cc}
\beta_{\bar{\beta}}^{\prime} & \beta_{\bar{\delta}}^{\prime} \\
\delta_{\bar{\beta}}^{\prime} & \delta_{\bar{\delta}}^{\prime}
\end{array}\right)\left(\begin{array}{cc}
\bar{\beta}_{\beta} & \bar{\beta}_{\delta} \\
\bar{\delta}_{\beta} & \bar{\delta}_{\delta}
\end{array}\right) \text {. }
$$

It has been shown that a consequence of (11) is that $\alpha^{\prime}{ }_{\alpha}=1$, whence

$$
\alpha^{\prime}=\alpha+\lambda^{\prime}(\beta, p, \delta) .
$$

Applying this relation to the two components of the system under consideration,

Thus

$$
\begin{aligned}
& \alpha^{\prime}=\bar{\alpha}+\bar{\lambda}(\bar{\beta}, \bar{p}, \bar{\delta}) \\
& \bar{\alpha}=\alpha+\lambda(\beta, p, \delta) .
\end{aligned}
$$

$$
\alpha^{\prime}-\alpha=\lambda^{\prime}=\bar{\lambda}+\lambda .
$$

Thus the geometrical properties of a rotationally-symmetric optical system can be described completely by the system of equations

and

$$
\alpha^{\prime}-\alpha=\sum_{i=1}^{n} \lambda_{i}
$$

$$
\left(\begin{array}{cc}
\beta_{\beta}^{\prime} & \beta_{\delta}^{\prime} \\
\delta_{\beta}^{\prime} & \delta_{\delta}^{\prime}
\end{array}\right)=\prod_{i=1}^{n}\left(\begin{array}{ll}
\partial \beta_{i} / \partial \beta_{i-1} & \partial \beta_{i} / \partial \delta_{i-1} \\
\partial \delta_{i} / \partial \beta_{i-1} & \partial \delta_{i} / \partial \delta_{i-1}
\end{array}\right),
$$

where $\lambda_{i+1}$ is a function of $\beta_{i}, p$ and $\delta_{i}$, where $\beta^{\prime}=\beta_{n}, \quad \delta^{\prime}=\delta_{n}$, and where $\beta=\beta_{0}, \delta=\delta_{0}$. This is a system of five scalar equations of which four are independent by (14).

If we replace the left members of these equations by functions representing a perfect optical system we are left with equations of condition on the parameter occurring in each of the components of the system. Alternatively a measure of the failure of the equations to be satisfied can be taken as a measure of the aberrations of the optical system.

\section{Functions for a Perfect Optical System.}

A possible choice for the variables, suitable for work with an optical system with object and image planes located a finite distance from the lens is

$$
\left.\begin{array}{l}
\alpha=\arctan y / x, \\
\beta=\sqrt{x^{2}+y^{2}}, \\
\delta=\frac{x \xi+y \eta}{\sqrt{x^{2}+y^{2}}}
\end{array}\right\}
$$

It can be verified that these satisfy (9) when $\gamma=p$.

By integrating the equations for a perfect optical system (1) and (2), we obtain $x^{\prime}=m x$, $y^{\prime}=m y$, the constant of integration being eliminated by a suitable choice of coordinate axes. 
From this

$$
\alpha^{\prime}=\alpha, \text { and } \beta^{\prime}=m \beta,
$$

whence $\beta^{\prime}{ }_{\beta}=m$ and $\beta_{\delta}^{\prime}=0$. Substituting this in (14) we obtain

$$
m \delta^{\prime}{ }_{\delta}=1 \text {. }
$$

Integrating:

$$
\delta^{\prime}=\delta / m+\psi,
$$

where $\psi$ is an arbitrary function of $\beta$. Thus for a perfect optical system described by the variables in $(25)$ we have

$$
\alpha^{\prime}-\alpha=\sum_{i} \lambda_{i}=0
$$

and

$$
\left(\begin{array}{cc}
m & 0 \\
\psi_{\beta} & 1 / m
\end{array}\right)=\Pi_{i}\left(\begin{array}{cc}
\partial \beta_{i} / \partial \beta_{i-1} & \partial \beta_{i} / \partial \delta_{i-1} \\
\partial \delta_{i} / \partial \beta_{i-1} & \partial \delta_{i} / \partial \delta_{i-1}
\end{array}\right)
$$

as our equations of condition. It can be shown that $\psi_{\beta}$ when $\beta=0$ equals the reciprocal of the focal length of the perfect lens.

The complexity of the problem may be reduced somewhat by the following considerations. Suppose (27) were satisfied for meridian rays only. Then

$$
\begin{aligned}
& \beta^{\prime}=m \beta+\epsilon_{1}(\beta, \delta, p) \\
& \delta^{\prime}=\delta / m+\epsilon_{2}(\beta, \delta, p)
\end{aligned}
$$

where $\epsilon_{1}=\epsilon_{2}=0$ when $p=0$. If (26) is subsequently corrected for skew rays then $\alpha^{\prime}{ }_{\beta}=\alpha^{\prime}{ }_{\delta}=0$, which by (15) implies that $\beta^{\prime}{ }_{p}=\delta^{\prime}{ }_{p}=0$. Thus the derivatives of $\epsilon_{1}$ and $\epsilon_{2}$ with respect to $p$ are zero. Therefore these two functions are independent of $p$. Since $\epsilon_{1}$ and $\epsilon_{2}$ vanish for a particular value of $p$, they must equal zero for all values and (27) is satisfied for skew rays as well as meridian rays. Thus, the solution of (27) for meridian rays and the solution of (26) for skew rays assures the correction of the optical system for skew rays.

\section{Transfer and Refraction Calculations}

The ultimate components of an optical system will be considered here to consist of (a) a transfer across a space between two planes and (b) a transfer from a plane (which will be called the reference plane) to a refracting surface, refraction across the surface and a transfer back to the reference plane. The ray tracing formulas for the transfer are

$$
\begin{aligned}
x^{\prime} & =x+t \xi / \zeta \\
y^{\prime} & =y+t \eta / \zeta \\
\xi^{\prime} & =\xi \\
\eta^{\prime} & =\eta
\end{aligned}
$$

where $t$ is the distance between the two planes, whence

$$
\begin{aligned}
\alpha^{\prime} & =\alpha+\arctan \frac{t p}{\beta^{2} \zeta+t \beta \delta}, \\
& =\alpha+\arctan p / \beta \delta-\arctan p / \beta^{\prime} \delta^{\prime}, \\
\beta^{\prime} & =\sqrt{(\beta+t \delta / \zeta)^{2}+(t p / \beta \zeta)^{2}}, \\
\delta^{\prime} & =\frac{\delta(\beta+t \delta / \zeta)+(t p / \beta \zeta) p / \beta}{\sqrt{(\beta+t \delta / \zeta)^{2}+(t p / \beta \zeta)^{2}}} .
\end{aligned}
$$


For meridian rays the latter two formulas are

$$
\left.\begin{array}{l}
\beta^{\prime}=(\beta-+t \delta / \zeta) \\
\delta^{\prime}=\delta,
\end{array}\right\}
$$

where $\zeta^{2}=N^{2}-\delta^{2}$. Accordingly the matrix representing transfer between two planes separated by a distance $t$, in a medium with index of refraction $N$, is

$$
m_{T}=\left(\begin{array}{cc}
1 & N^{2} t / \xi^{3} \\
0 & 1
\end{array}\right) .
$$

The ray tracing formulas for a transfer from a plane to a refracting surface, refraction across the surface, and a transfer from the surface back to the plane are, respectively

$$
\left.\begin{array}{l}
\bar{x}=x+\bar{z} \xi / \zeta \\
\bar{y}=y+\bar{z} \eta \zeta, \\
\xi^{\prime}=\xi+\bar{x} \phi \\
\eta^{\prime}=\eta+\bar{y} \phi \\
\zeta^{\prime}=\zeta-\bar{\beta} \phi / \bar{z} \bar{\beta} \\
x^{\prime}=\bar{x}-\bar{z} \xi^{\prime} / \zeta^{\prime} \\
y^{\prime}=\bar{y}-\bar{z} \xi^{\prime} / \zeta^{\prime},
\end{array}\right\}
$$

where $\bar{z}$ is a function of $\bar{\beta}$ denoting the refracting surface and where

$$
\phi=\frac{\bar{z} \bar{\beta}}{\bar{\beta}\left(1+\bar{z}_{\bar{\beta}}^{2}\right)}\left\{(\zeta-\bar{\delta} \bar{z} \bar{\beta})-\sqrt{\left(\zeta-\bar{\delta} \bar{z}_{\bar{\beta}}\right)^{2}-\left(1+\bar{z}_{\bar{\beta}}^{2}\right)\left(N^{2}-N^{\prime 2}\right)}\right\} .
$$

In terms of the $\alpha, \beta, \delta$ coordinate system, these are

$$
\left.\begin{array}{c}
\bar{\alpha}=\alpha+\arctan \frac{\bar{z} p}{\beta^{2} \zeta+\bar{z} \beta \delta}, \\
\bar{\beta}=\sqrt{(\beta+\bar{z} \delta / \zeta)^{2}+(\bar{z} p / \beta \zeta)^{2}}, \\
\bar{\delta}=\frac{\delta(\beta+\bar{z} \delta / \zeta)+(p / \beta)(\bar{z} p / \beta \zeta)}{\sqrt{(\beta+\bar{z} \delta / \zeta)^{2}+(\bar{z} p / \beta \zeta)^{2}}},
\end{array}\right\}
$$


Thus for skew rays

$$
\begin{aligned}
& \alpha^{\prime}=\alpha-\arctan \frac{p}{\beta^{\prime} \delta^{\prime}}+\arctan \frac{p}{\bar{\beta} \bar{\delta}^{\prime}}-\arctan \frac{p}{\beta \delta}+\arctan \frac{p}{\beta \delta} \\
& =\alpha-\arctan \frac{p}{\beta^{\prime} \delta^{\prime}}+\arctan \frac{p \bar{\beta}\left(\bar{\delta}-\bar{\delta}^{\prime}\right)}{\bar{\beta}^{2} \bar{\delta} \bar{\delta} \bar{\delta}^{\prime}+p^{2}}+\arctan \frac{p}{\beta \delta} \\
& =\alpha-\arctan \frac{p}{\beta^{\prime} \delta^{\prime}}-\arctan \frac{p \phi}{\bar{\beta} \bar{\delta} \phi+\left(\bar{\delta}^{2}+p^{2} / \bar{\beta}^{2}\right)}+\arctan \frac{p}{\beta \delta} .
\end{aligned}
$$

For meridian rays the formulas for $\beta$ and $\delta$ reduce from those given in (33), (34), and (35) to

$$
\left.\begin{array}{l}
\bar{\beta}=\beta+\bar{z} \delta / \zeta \\
\bar{\delta}=\delta
\end{array}\right\}
$$

and

$$
\left.\begin{array}{l}
\beta^{\prime}=\overline{\beta^{\prime}}-\bar{z} \bar{\delta}^{\prime} / \zeta^{\prime} \\
\delta^{\prime}=\overline{\delta^{\prime}}
\end{array}\right\}
$$

where $\zeta^{2}=N^{2}-\delta^{2}$ and $\zeta^{\prime 2}=N^{\prime 2}-\delta^{\prime 2}$. of $\bar{\beta}$, we obtain

By differentiating the first equation of (37) with respect to $\beta$ and $\delta$, recalling that $\bar{z}$ is a function

$$
\begin{aligned}
& \bar{\beta}_{\beta}=1+\bar{z}_{\bar{\beta}} \bar{z}_{\beta} \delta / \zeta \\
& \bar{\beta}_{\hat{o}}=\bar{z} / \zeta+\bar{z}_{\bar{\beta}}-\bar{\beta}_{\delta} \delta / \zeta+\bar{z} \delta^{2} / \zeta^{3}
\end{aligned}
$$

so that

$$
\begin{aligned}
& \bar{\beta}_{\beta}=\zeta / \Delta \\
& \bar{\beta}_{\delta}=(\zeta / \Delta)\left(N^{2} \bar{z} / \zeta^{3}\right),
\end{aligned}
$$

where

$$
\Delta=\zeta-\bar{z} \bar{\beta} \bar{\delta}
$$

Thus

$$
\begin{aligned}
m_{a}=\left(\begin{array}{cc}
\bar{\beta}_{\beta} \bar{\beta}_{\delta} \bar{\delta}_{\delta} \\
\delta_{\beta} \delta_{\delta}
\end{array}\right) & =\left(\begin{array}{cc}
\zeta / \Delta & (\zeta / \Delta)\left(N^{2} \bar{z} / \zeta^{3}\right) \\
0 & 1
\end{array}\right) \\
& =\left(\begin{array}{cc}
\zeta / \Delta & 0 \\
0 & 1
\end{array}\right)\left(\begin{array}{cc}
1 & N^{2} \bar{z} / \zeta^{3} \\
0 & 1
\end{array}\right)
\end{aligned}
$$

Ev differentiating the equations of (38),

$$
m_{b}=\left(\begin{array}{cc}
\bar{\beta}^{\prime}{ }_{\bar{\beta}} & \bar{\beta}^{\prime}{ }_{\bar{\delta}} \\
\overline{\delta^{\prime}}{ }_{\bar{\beta}} & \bar{\delta}^{\prime}{ }_{\bar{\beta}}
\end{array}\right)=\left(\begin{array}{cc}
1 & 0 \\
\bar{\beta} \zeta^{\prime} \bar{z} \overline{\beta \beta} \phi / \bar{z} \bar{\beta} \Delta^{\prime} & 1+\bar{\beta} \phi\left(\bar{\delta}+\overline{z_{\beta} \zeta}\right) / \Delta^{\prime} \zeta
\end{array}\right),
$$

where $\Delta^{\prime}=\zeta^{\prime}-\bar{z}_{\bar{\beta}} \bar{\delta}^{\prime}$. From the definitions of $\Delta$ and $\Delta^{\prime}$ and from (34) it can be shown that $\left.1+\bar{\beta} \phi(\bar{\delta}+\bar{z} \bar{\beta} \zeta) / \Delta^{\prime} \zeta=\Delta \zeta^{\prime}\right) / \Delta^{\prime} \zeta$, resulting in a simplification of $m_{b}$. 
Again, differentiating (39) we obtain

$$
\begin{aligned}
m_{c} & =\left(\begin{array}{cc}
\beta^{\prime} \bar{\beta}^{\prime} & \beta^{\prime} \bar{\delta}^{\prime} \\
\delta^{\prime} \bar{\beta}^{\prime} & \delta^{\prime} \bar{\delta}^{\prime}
\end{array}\right)=\left(\begin{array}{cc}
\Delta^{\prime} / \zeta^{\prime} & -\bar{z} N^{\prime 2} / \zeta^{\prime 3} \\
0 & 1
\end{array}\right) \\
& =\left(\begin{array}{cc}
1 & -\bar{z} N^{\prime 2} / \zeta^{\prime 3} \\
0 & 1
\end{array}\right)\left(\begin{array}{cc}
\Delta^{\prime} / \zeta^{\prime} & 0 \\
0 & 1
\end{array}\right) .
\end{aligned}
$$

When these three matrices are multiplied together, we have

$$
\begin{aligned}
m_{R} & =m_{c} m_{b} m_{a} \\
& =\left(\begin{array}{cc}
1 & -\bar{z} N^{\prime 2} / \zeta^{\prime 3} \\
0 & 1
\end{array}\right)\left(\begin{array}{lc}
\Delta^{\prime} \zeta / \Delta \zeta^{\prime} & 0 \\
\bar{\beta} \zeta \zeta^{\prime} \bar{z} \overline{\beta \beta} \phi / \bar{z} \bar{\beta} \Delta \Delta^{\prime} & \Delta \zeta^{\prime} / \Delta^{\prime} \zeta
\end{array}\right)\left(\begin{array}{cc}
1 & \dot{z} N^{2} / \zeta^{3} \\
0 & 1
\end{array}\right),
\end{aligned}
$$

Note that the first and last factors are formally identical to $m_{T}$ and can therefore be combined conveniently with an adjoining transfer matrix.

When the refracting surface is a sphere centered on the reference plane

$$
\bar{z}=-\frac{1}{c} \sqrt{1-c^{2} \bar{\beta}^{2}}
$$

The minus sign assures that the sphere is concave to the right when $c$ is positive, in accordance with the usual convention. Then

$$
m_{R}=\left(\begin{array}{cc}
1 & -\bar{z} N^{\prime 2} / \zeta^{\prime 3} \\
0 & 1
\end{array}\right)\left(\begin{array}{ll}
\Delta^{\prime} \zeta / \Delta \zeta^{\prime} & 0 \\
\zeta \zeta^{\prime} \phi / c^{2} \bar{z}^{2} \Delta \Delta^{\prime} & \Delta \zeta^{\prime} / \Delta^{\prime} \zeta
\end{array}\right)\left(\begin{array}{cc}
1 & \bar{z} N^{2} / \zeta^{3} \\
0 & 1
\end{array}\right)
$$

where

$$
\left.\begin{array}{rl}
\Delta & =(\bar{\beta} \bar{\delta}+\bar{z} \zeta) / \bar{z} \\
\Delta^{\prime} & =\left(\bar{\beta} \bar{\delta}^{\prime}+\bar{z} \zeta^{\prime}\right) / \bar{z}=\sqrt{\Delta^{2}-\frac{1}{c^{2} \bar{z}}\left(N^{2}-N^{\prime 2}\right)}, \\
\phi & =-c^{2} \bar{z}\left(\Delta-\Delta^{\prime}\right) .
\end{array}\right\}
$$

\section{A Simple Illustration}

As an example we consider the properties of a single refracting spherical surface. Consider an optical system consisting of an object plane, a spherical surface separating media of refractive indices $N$ and $N^{\prime}$ and an image plane. Let the object and image planes be located distances $t$ and $t^{\prime}$ from the center of the spherical surface. Figure 1 shows the case where $t$,

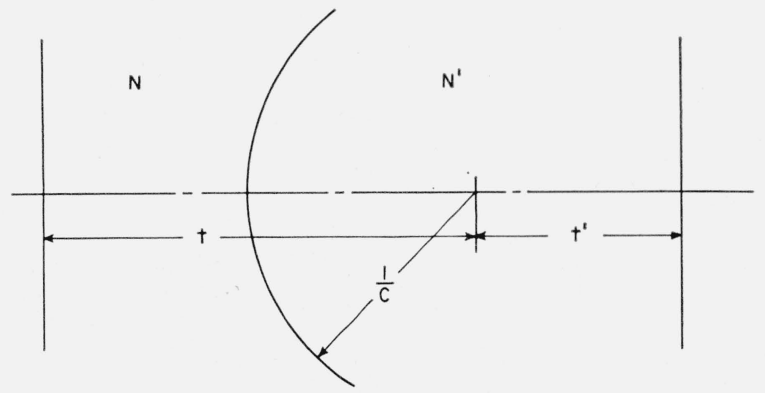

FIGURE 1 
$t^{\prime}$, and $c$ are positive. From (27), (30), and (41) the equation of condition for meridian rays is

$$
\begin{aligned}
& \left(\begin{array}{cc}
m & 0 \\
\psi_{\beta} & 1 / m
\end{array}\right) \\
& \quad=\left(\begin{array}{cc}
1 & N^{\prime 2} t^{\prime} / \zeta^{\prime 3} \\
0 & 1
\end{array}\right)\left(\begin{array}{cc}
1 & -\bar{z} N^{\prime 2} / \zeta^{\prime 3} \\
0 & 1
\end{array}\right)\left(\begin{array}{cc}
\Delta^{\prime} \zeta / \Delta \zeta^{\prime} & 0 \\
\zeta \zeta^{\prime} \phi / c^{2} \bar{z}^{2} \Delta \Delta^{\prime} & \Delta \zeta^{\prime} / \Delta^{\prime} \zeta
\end{array}\right)\left(\begin{array}{cc}
1 & \bar{z} N^{2} / \zeta^{3} \\
0 & 1
\end{array}\right)\left(\begin{array}{cc}
1 & N^{2} t / \zeta^{3} \\
0 & 1
\end{array}\right)
\end{aligned}
$$

For skew rays, by (26), (28), and (36),

$$
\alpha^{\prime}-\alpha=-\arctan \frac{p}{\beta^{\prime} \delta^{\prime}}+\arctan \frac{p}{\beta \delta}-\arctan \frac{p \phi}{\overline{\beta \delta} \phi+\left(\overline{\delta^{2}}+p^{2} / \bar{\beta}^{2}\right)}=0 .
$$

Expressing (43) in scalar form we obtain four equations.

$$
\left.\begin{array}{r}
\Delta^{\prime} \zeta / \Delta \zeta^{\prime}+N^{\prime 2}\left(t^{\prime}-\bar{z}\right) \zeta \phi / c^{2} \bar{z}^{2} \Delta \Delta^{\prime} \zeta^{\prime 2}=m \\
\zeta \zeta^{\prime} \phi / c^{2} \bar{z}^{2} \Delta \Delta^{\prime}=\psi_{\beta} \\
\frac{N^{2}(t+\bar{z}) \Delta^{\prime}}{\Delta \zeta^{2} \zeta^{\prime}}+\frac{N^{\prime 2}\left(t^{\prime}-\bar{z}\right) \Delta}{\Delta^{\prime} \zeta \zeta^{\prime 2}}+\frac{N^{2}(t+\bar{z}) N^{\prime 2}\left(t^{\prime}-\bar{z}\right) \phi}{c^{2} \bar{z}^{2} \Delta \Delta^{\prime} \zeta^{2} \zeta^{\prime 2}}=0 \\
\Delta \zeta^{\prime} / \Delta^{\prime} \zeta+N^{2}(t+\bar{z}) \zeta^{\prime} \phi / c^{2} \bar{z}^{2} \Delta \Delta^{\prime} \zeta^{2}=1 / m .
\end{array}\right\}
$$

We will pay particular attention to the third of these, which we write in the form

$$
N^{2}(t+\bar{z}) \Delta^{\prime 2} \zeta^{\prime}+N^{\prime 2}\left(t^{\prime}-\bar{z}\right) \Delta^{2} \zeta+\left(1 / c^{2} \bar{z}^{2}\right)(t+\bar{z}) N^{\prime 2}\left(t^{\prime}-z\right) \phi=0 .
$$

Let

$$
\begin{aligned}
\sigma & =\Delta \bar{z}=\beta \delta+\bar{z} \zeta, \\
\sigma^{\prime} & =\Delta^{\prime} \bar{z}=\beta \delta^{\prime}+\bar{z} \zeta^{\prime}=\sqrt{\sigma^{2}-\left(1 / c^{2}\right)\left(N^{2}-N^{\prime 2}\right)} .
\end{aligned}
$$

Also,

$$
\zeta^{\prime}=\zeta+\bar{z} \phi
$$

where $\phi=-c^{2}\left(\sigma-\sigma^{\prime}\right)$ by (42). Substituting these yields

$$
c^{2} \sigma^{2} \zeta\left[N^{\prime 2}\left(t^{\prime}-\bar{z}\right)+N^{2}(t+\bar{z})\right]-N^{2} \zeta(t+\bar{z})\left(N^{2}-N^{\prime 2}\right)+N^{2}(t+\bar{z})\left[c^{2} \sigma^{2} \bar{z}-N^{2} \bar{z}+N^{\prime 2} t^{\prime}\right] \phi=0 .
$$

This may be written as

$$
c^{2} \sigma^{2} \zeta\left(N^{\prime 2} t^{\prime}+N^{2} t\right)+\zeta\left(N^{2}-N^{\prime 2}\right)\left[c^{2} \sigma^{2} \bar{z}-N^{2}(t+\bar{z})\right]+N^{2}(t+\bar{z})\left[c^{2} \sigma^{2} \bar{z}-N^{2} \bar{z}+N^{\prime 2} t^{\prime}\right] \phi=0 .
$$

If

$$
N^{\prime 2} t^{\prime}+N^{2} t=0
$$

Then

$$
\left[\zeta\left(N^{2}-N^{\prime 2}\right)+N^{2}(t+\bar{z}) \phi\right]\left[c^{2} \sigma^{2} \bar{z}-N^{2}(t+\bar{z})\right]=0 .
$$

We thus obtain two possible cases represented by the two equations

$$
\begin{gathered}
\zeta\left(N^{2}-N^{\prime 2}\right)+N^{2}(t+\bar{z}) \phi=0 \\
c^{2} \sigma^{2} \bar{z}-N^{2}(t+\bar{z})=0 .
\end{gathered}
$$


When $\bar{\beta}=\bar{\delta}=0$,

$$
\bar{z}=-1 / c, \sigma=-N / c, \sigma^{\prime}=-N^{\prime} / c, \zeta=N \text {, and } \phi=c\left(N-N^{\prime}\right) .
$$

Substituting in (48) we obtain

$$
\begin{gathered}
N\left(N^{2}-N^{\prime 2}\right)+N^{2}(t-1 / c) c\left(N-N^{\prime}\right)=0, \\
N+N^{\prime}+N(c t-1)=0, \\
t=-N^{\prime} / N c .
\end{gathered}
$$

Applying this to (47),

$$
t^{\prime}=N / N^{\prime} c .
$$

When $\bar{\beta}$ and $\bar{\delta}$ are not zero, it can be shown that (50) and (51) satisfy (48) for all values of $\delta$ provided that $\beta=0$. Therefore only the axial point on the object plane is imaged perfectly. This result provides the aplanatic points of a sphere [9] (see fig. 2).

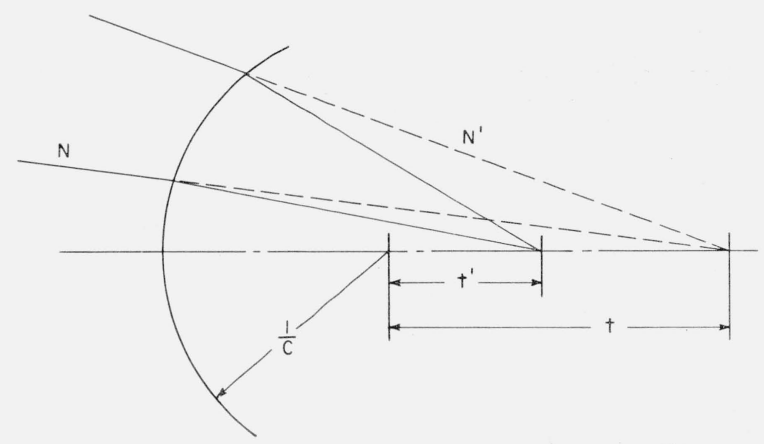

FIGURE 2

On making the same substitutions into (49) we find that $t=0$, which when applied to $(47)$ yields $t^{\prime}=0$. As in the previous case, these are true for all values of $\bar{\beta}$ and $\bar{\delta}$ provided that $\beta=0$. This illustrates the well-known fact that the center of a sphere is imaged perfectly on itself [4].

The author acknowledges with thanks the help and encouragement of Dr. W. T. Welford, under whose supervision much of this work was done at Imperial College of Science and Technology, London.

\section{References}

[1] M. Herzberger, Trans. Am. Math. Soc. 53, 21 (1943).

[2] R. Straubel, Physik. Z. 4, 114 (1902).

[3] G. Toraldo di Francia, J. Opt. Soc. Am. 40, 600 (1950).

[4] M. Herzberger, Modern geometrical optics, p. 21 (Interscience Publishers Inc., New York, N.Y., 1958).

Washington, D.C.

(Paper 63B1-6) 